\title{
Avaliação de um método de estimativa de idade pela mineralização dentária dos terceiros molares
}

\author{
Evaluation of an age estimate method by dental mineralization of third molars \\ Evaluación de um método de estimación de edad por la mineralización dental de los terceiros
} molares

\author{
Nicole Paiva Veras \\ ORCID: https://orcid.org/0000-0002-5719-5983 \\ Universidade Federal do Maranhão, Brasil \\ E-mail: npaivaveras@gmail.com \\ César Augusto Abreu-Pereira \\ ORCID: https://orcid.org/0000-0003-0665-9060 \\ Universidade Estadual Paulista, Brasil \\ E-mail: cesar.abreu@unesp.br \\ Priscila Letícia Vieira Kitagawa \\ ORCID: https://orcid.org/0000-0002-7779-043X \\ Faculdade de Odontologia de Ribeirão Preto, Brasil \\ E-mail: kitagawa.priscila@gmail.com \\ Maria Aparecida Costa \\ ORCID: https://orcid.org/0000-0001-6349-9206 \\ Instituto Federal do Maranhão, Brasi \\ E-mail: mapa.costa@uol.com.br \\ Laise Nascimento Correia Lima \\ ORCID: https://orcid.org/0000-0002-6871-3491 \\ Universidade Federal da Paraíba, Brasil \\ E-mail: laiselima@gmail.com \\ José Ferreira Costa \\ ORCID: https://orcid.org/0000-0002-6134-5323 \\ Universidade Federal do Maranhão, Brasil \\ E-mail: jf.costa@ufma.br \\ Rosana Costa Casanovas \\ ORCID: https://orcid.org/0000-0002-6871-3491 \\ Universidade Federal do Maranhão, Brasil \\ E-mail: rosana.c.casanovas@hotmail.com
}

\begin{abstract}
Resumo
A Odontologia Legal utiliza a mineralização dentária na estimativa da idade, para auxiliar na estimativa de cadáveres e até mesmo de humanos vivos. O objetivo do estudo foi avaliar a confiabilidade do método proposto por Cornélio Neto (2000) para estimar a idade cronológica humana por meio da análise da mineralização dos terceiros molares. Foram utilizadas 150 radiografias panorâmicas de indivíduos de ambos os sexos, com faixa etária entre 15 e 22 anos e que possuíssem, pelo menos, um dos terceiros molares. Os estágios de mineralização de cada elemento presente na radiografia foram registrados, de acordo com a classificação de Nicodemo, Moraes e Médici Filho (1974) e adaptada por Cornélio Neto (2000), por um examinador previamente calibrado. Com a determinação dos estágios de mineralização foram aplicados nas fórmulas desenvolvidas por Cornélio Neto (2000) para estimativa da idade, que varia de acordo com cada um dos terceiros molares e o sexo dos indivíduos. Para a análise de dados, foi realizado o teste de Coeficiente de Correlação Intraclasse, utilizando o software SPSS (Versão 27, Chicago, EUA). No sexo masculino o dente com maior taxa de concordância foi o dezoito, já no sexo feminino foram os dentes trinta e oito e quarenta e oito. A classificação da concordância, proposta por Fermanian (1984), aponta que os terceiros molares avaliados possuem correlação moderada entre as idades reais e estimadas. Sendo assim, este método pode ser usado como um exame complementar para estimar a idade real de um indivíduo, necessitando de outros que corroborem com sua estimativa.
\end{abstract}

Palavras-chave: Determinação da idade pelos dentes; Radiografia dentária; Odontologia legal.

\section{Abstract}

Forensic Dentistry uses dental mineralization to estimate age, in order to assist the estimation of cadavers and also living humans. The purpose of the study was to evaluate the reliability of the method proposed by Cornélio Neto (2000) to estimate human chronological age by analyzing third molars mineralization. The study used 150 panoramic 
X-rays of people from both sexes, on the age between 15 and 22 years and who had at least one of the third molars. According to the classification in Nicodemo, Moraes and Médici Filho (1974) and adapted by Cornélio Neto (2000), the mineralization stages of each element present on the radiography were recorded by a previously calibrated examiner. After the mineralization stages were stated, they were intented in the formulas developed by Cornélio Neto (2000) to estimate age, which varies according to each of the third molars and the sex of the owners. For data analysis, the Intraclass Correlation Coefficient test was performed, using the SPSS software (Version 27, Chicago, USA). In males, the tooth with the highest agreement rate was the eighteen, whereas in females, teeth were thirtyeight and forty-eight. The classification of agreement, proposed by Fermanian (1984), points out that the assessed third molars had a moderate correlation between real and estimated ages. Therefore, this method can be used as a complementary exam to estimate an individual's real age, requiring other studies to corroborate his estimate.

Keywords: Age determination by teeth; Radiography, Dental; Forensic dentistry.

\section{Resumen}

La Odontología Legal utiliza la mineralización dental en la estimación de la edad, para auxiliar en la estimación de cadáveres e incluso de humanos vivos. El objetivo del estudio fue evaluar la confiabilidad del método propuesto por Cornélio Neto (2000) para estimar la edad cronológica humana por medio del análisis de la mineralización de los terceros molares. Fueron utilizadas 150 radiografías panorámicas de individuos de ambos sexos, con rango de edad entre 15 y 22 años y que poseen, por lo menos, uno de los terceros molares. Las etapas de mineralización de cada elemento presente en la radiografía fueron registradas, de acuerdo con la clasificación en Nicodemo, Moraes y Médici Filho (1974) y adaptada por Cornélio Neto (2000), con un examinador previamente calibrado. La determinación de las etapas de mineralización fue aplicada en las fórmulas desarrolladas por Cornélio Neto (2000) para estimación de la edad, que varía de acuerdo con cada uno de los terceros molares y el sexo de los indivíduos. Para el análisis de los datos, fue realizado el test de Coeficiente de Correlación Intraclase, utilizando el software SPSS (Versión 27, Chicago, EUA). En el sexo masculino el diente con mayor tasa de concordancia fue el dieciocho (18), ya en el sexo femenino fueron los dientes treinta y ocho (38) y cuarenta y ocho (48). La clasificación de la concordancia, propuesta por Fermanian (1984), apunta a que los terceros molares evaluados poseen correlación moderada entre las edades reales y estimadas. Siendo así, este método puede ser usado como un examen complementario para estimar la edad real de un individuo, necesitando de otros que corroboren con su estimación.

Palabras clave: Determinación de la edad por los dientes; Radiografía dental; Odontología legal.

\section{Introdução}

A mineralização de terceiros molares vem sendo utilizada como recurso na Odontologia Forense, servindo de parâmetro para auxiliar na estimativa da idade de cadáveres não identificados, e até mesmo de humanos vivos (Dhanjal et al., 2006; Orhan et al., 2007; Lee et al., 2009; Gioster-Ramos et al., 2021). Esse tipo de estimativa de idade tem colaborado com investigações criminais. Assim, muitos jovens infratores, quando detidos em flagrante, simulam uma idade inferior a maioridade penal para que não recebam as penalidades previstas no Código Penal. Com isso, é necessário que a autoridade policial encaminhe este indivíduo para perícia de estimativa da idade nas Perícias Oficiais Criminais (Cornélio-Neto et al., 2006).

Existem vários métodos para estimativa de idade, sendo a maioria baseada em parâmetros anatômicos ósseos ou mineralização dos dentes (Carvalho, 1990; Oliveira, 2010). No Brasil, Nicodemo (1967) fez um estudo pioneiro com o objetivo de avaliar a cronologia da mineralização dos terceiros molares em moradores do Vale do Paraíba, no estado de São Paulo. O autor observou que nenhuma pesquisa se aplicava às características da população brasileira e utilizou o método radiográfico para estimar a idade de 215 indivíduos e comprovar a confiabilidade do seu estudo.

O estudo de Nascimento et al. (2020) propôs avaliar a aplicabilidade do método da medição de ápices abertos proposto por Cameriere et al. (2006), numa população de nordestinos em João Pessoa, Paraíba. Os resultados desse estudo mostraram que o método é aplicável às pessoas com idade entre 5 e 14 anos, já que a média entre a idade estimada e a idade cronológica real foi próxima a zero. Porém, o método não é aplicável a indivíduos de qualquer idade, necessitando de outras abordagens para uma estimativa mais fiel.

O método radiográfico é um dos mais utilizados para realização de estimativa de idade, pois além de ser de baixo custo, é prático e de fácil acesso, além de independer da erupção dentária (Moreno et al., 2014) Os terceiros molares são os 
únicos dentes que continuam com sua mineralização até depois dos 21 anos, sendo elementos de eleição na estimativa de idade (Cornélio-Neto et al., 2006).

Uma grande contribuição para a validação de métodos estimativa de idade através de exame radiográfico foi o trabalho desenvolvido por Nolla em 1960, onde foi proposto uma tabela de avaliação da idade dentária, criada a partir de uma amostra de 25 pacientes do sexo feminino e 25 do sexo masculino, em Michigan (EUA). Para isso, o desenvolvimento dos dentes permanentes foi classificado por meio de radiografias intra e extrabucais, e dividiu a mineralização em 11 fases, onde 0Ausência de esboço ou cripta; 1- Presença de esboço; 2- Início da mineralização; 3- 1/3 da coroa formada; 4- 2/3 da coroa formada; 5- Coroa Formada; 6- Início radicular; 7- 1/3 da raiz formada; 8- 2/3 da raiz formada; 9- Raiz incompleta e 10 - Raiz completa.

Cornélio-Neto (2000) dedicou seu estudo para obter uma equação de cada um dos quatro terceiros molares no qual ao analisar a fase de mineralização proposta por Nicodemo, Moraes e Médici Filho (1974) e adaptada pelo autor para aumentar a margem de aproximação da estimativa da idade, colocaria a fase como variável numa fórmula e assim poderia estimar a idade dos indivíduos em meses. Para isso, ele avaliou crianças e jovens entre 5 e 22 anos, a partir de 211 radiografias panorâmicas, analisando o grupo de terceiros molares.

Diante da grande importância do tema, o objetivo deste trabalho foi avaliar a aplicabilidade e confiabilidade do método de equação proposto por Cornélio em 2000 para estimar a maioridade penal e assim estabelecer corretamente deveres e direitos penais e civis dos cidadãos.

\section{Metodologia}

\subsection{Aspectos éticos}

Conforme recomendação da Resolução no . 466/12 do Conselho Nacional de Saúde do Ministério da Saúde, foram observados os aspectos éticos no que diz respeito à pesquisa envolvendo seres humanos no Brasil. O presente estudo de pesquisa foi encaminhado ao Comitê de Ética em Pesquisa da Universidade Federal do Maranhão e aprovado sob protocolo $n^{\circ}$ $620.034 / 2014$.

\subsection{Calibração do examinador}

O examinador foi previamente calibrado e este momento foi dividido em três ocasiões: I- o examinador observou, de forma cega e randomizada, radiografias panorâmicas previamente selecionadas, atribuindo um estágio de mineralização para cada terceiro molar; II- Após 15 dias, as radiografias foram reavaliadas para se obter a concordância intra-examinador; III- em um terceiro momento, foi realizado um ensaio com o número mínimo de 10 radiografias, onde foram testadas a aplicação dos estágios de mineralização nas fórmulas proposta por Cornélio Neto (2000).

\subsection{Amostras}

Foram utilizadas 150 radiografias panorâmicas de indivíduos de ambos os sexos, obtidas dos arquivos de prontuários de pacientes, sendo 150 provenientes de uma clínica de Radiologia da cidade de João Pessoa (Paraíba, Brasil), previamente autorizadas. Todas as radiografias foram realizadas em aparelho de RX panorâmico de mesma marca e com mesma intensidade de radiação. Foi utilizada a mesma marca de filme em todas as radiografias. As películas foram reveladas manualmente pelo método tempo e temperatura (TOSONI et al., 2003). Realizou-se a interpretação radiográfica em um aparelho negatoscópio da marca Línea 2000. 


\subsection{Critérios de inclusão e exclusão das amostras}

Foram incluídas as radiografias de pacientes que apresentaram faixa etária de 15 a 22 anos, na época da tomada radiográfica, e que possuíssem pelo menos um dos terceiros molares. As radiografias de má qualidade que apresentaram pouca nitidez, contraste ou visibilidade reduzida foram descartadas pelo examinador previamente calibrado, sendo este os critérios de exclusão deste estudo.

\subsection{Determinação do estágio de mineralização dos terceiros molares}

Os estágios de mineralização dos terceiros molares superiores e inferiores de cada indivíduo foram registrados, de acordo com a classificação proposta por Nicodemo, Moraes e Médici Filho (1974) por um examinador previamente calibrado, que não teve acesso prévio às informações relativas a cada indivíduo. Para isso, cada terceiro molar foi avaliado individualmente e um estágio de mineralização foi determinado de acordo com a sua fase anatômica de formação. Figura 1.

Figura 1. Estágios da mineralização dentária segundo a classificação de Nicodemo, Moraes e Médici Filho (1974). 0Ausência de esboço ou cripta. 1-Presença de esboço; 2- Início da mineralização; 3 - 1/3 da coroa formada; 4 - 2/3 da coroa formada; 5 - Coroa Formada; 6 - Início radicular; 7 - 1/3 da raiz formada; 8 - 2/3 da raiz formada; 9 - Raiz incompleta e 10 Raiz completa.

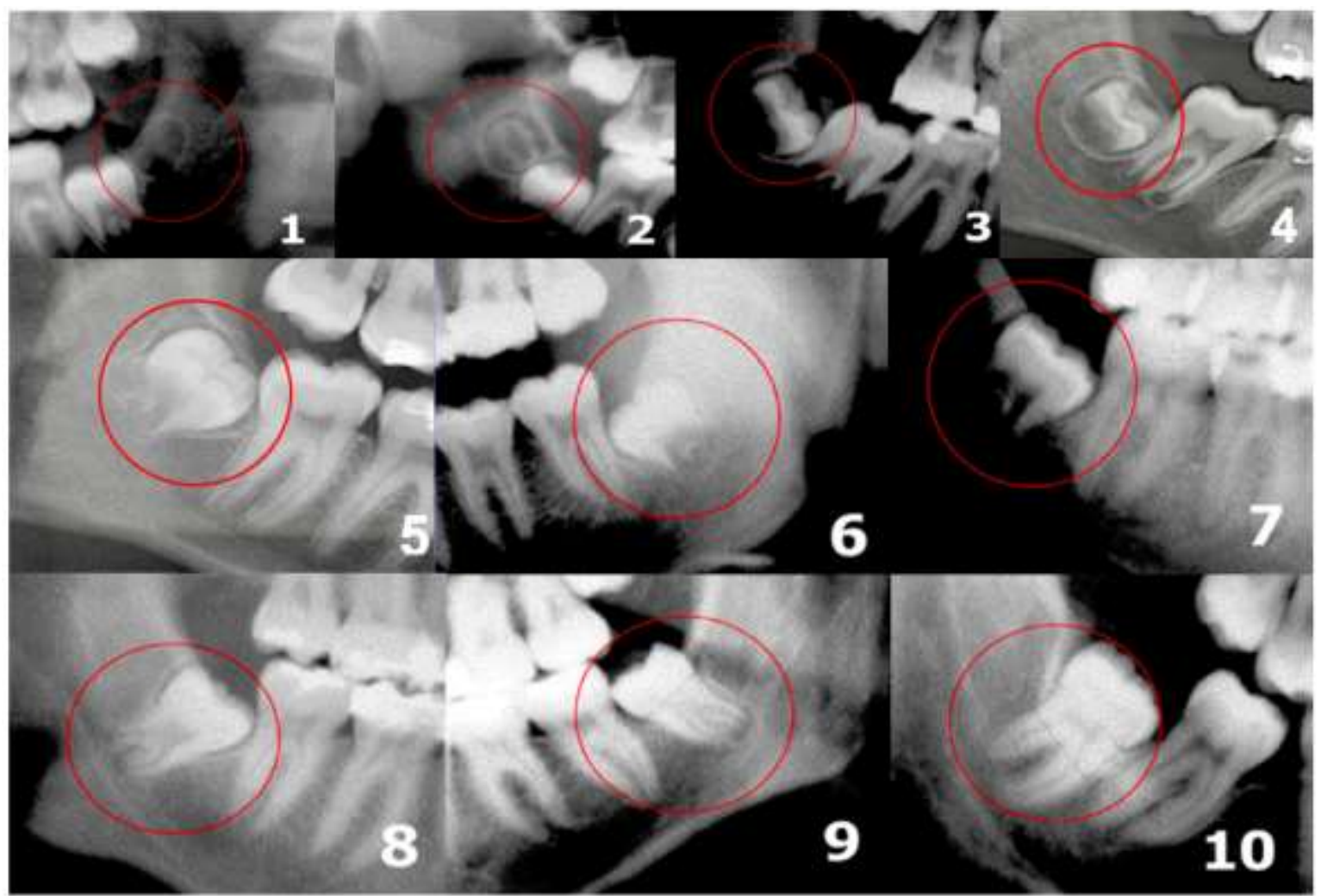

Fonte: Autores (2021).

\subsection{Aplicação da fórmula para estimativa de idade}

Os dados obtidos na determinação dos estágios de mineralização foram aplicados nas fórmulas (Tabela 1) desenvolvidas por Cornélio Neto (2000) para estimativa da idade, de acordo com cada um dos terceiros molares e o sexo dos indivíduos. 
Tabela 1: Fórmulas propostas por Cornélio Neto (2000) para estimar a idade de acordo com o sexo e o terceiro molar, onde Y corresponde à idade estimada do indivíduo e X a variável do estágio de mineralização em que se encontra o terceiro molar.

\begin{tabular}{ccc}
\hline Dente & Masculino & Feminino \\
\hline 18 & $\mathrm{Y}=84,85867+16,06224 . \mathrm{X}$ & $\mathrm{Y}=97,31289+14,07433 . \mathrm{X}$ \\
28 & $\mathrm{Y}=85,90333+16,11267 . \mathrm{X}$ & $\mathrm{Y}=102,801+13,32867 . \mathrm{X}$ \\
38 & $\mathrm{Y}=86,882+15,66818 . \mathrm{X}$ & $\mathrm{Y}=97,092+14,90745 . \mathrm{X}$ \\
48 & $\mathrm{Y}=87,518+15,64891 . \mathrm{X}$ & $\mathrm{Y}=103,49467+14,03206 . \mathrm{X}$ \\
\hline
\end{tabular}

Fonte: Cornélio Neto (2000).

Por fim, as idades estimadas foram contrastadas com a idade real dos pacientes presente nas radiografias que, até então, estavam ocultadas para caracterizar o estudo cego e diminuir a chance de indução de resultados na tabulação dos dados.

\subsection{Análise estatística dos dados}

Foi realizada toda a estatística descritiva dos dados, onde foi observado o coeficiente de variação, assimetria, curtose e possíveis outliers dos grupos. Após atender aos pressupostos, por se tratar de uma amostra pareada com dados quantitativos, foi realizado o teste de Coeficiente de Correlação Intraclasse, utilizando o software SPSS (Versão 27, Chicago, EUA). O teste foi realizado para cada dente $(18,28,38$ e 48$)$ e de acordo com o sexo (masculino e feminino), levando em consideração a idade estimada pela fórmula de Cornélio-Neto (2000) e a idade real de cada indivíduo, com nível de confiança de $95 \%$ ( $\alpha=$ 0,05). Por fim, foi feita a representação gráfica através do modelo de Bland e Altman (1983).

\section{Resultados}

Foram analisadas 150 radiografias panorâmicas de 50 (33\%) indivíduos do sexo masculino e 100 (67\%) indivíduos do sexo feminino. No sexo masculino, compreendiam 43 elementos 18, 44 elementos 28,48 elementos 38 e 46 elementos 48 . Já no sexo feminino, tinham 98 elementos 18, 99 elementos 28, 95 elementos 38 e 93 elementos 48 . Levando em consideração que foram feitas 3 análises da estimativa de idade, em três momentos distintos e depois extraída uma média, foram observados os valores dos dados médios (Average Measures) do output do teste de Coeficiente de Correlação intraclasse, conforme demonstrado na Tabela 2.

Tabela 2. Concordância para cada dente de acordo com o sexo $(\alpha=0,05)$.

\begin{tabular}{ccc}
\hline Dente & Masculino & Feminino \\
\hline 18 & 0,797 & 0,526 \\
28 & 0,711 & 0,555 \\
38 & 0,694 & 0,656 \\
48 & 0,686 & 0,651 \\
\hline
\end{tabular}

Fonte: Autores (2021).

Os resultados obtidos no Teste de Coeficiente de Correlação Intraclasse podem ser interpretados através da classificação proposta por Fermanian (1984), disponíveis na Tabela 3 e Figura 2. 
Research, Society and Development, v. 10, n. 7, e19410716524, 2021

(CC BY 4.0) | ISSN 2525-3409 | DOI: http://dx.doi.org/10.33448/rsd-v10i7.16524

Tabela 3. Classificação da concordância, segundo proposta de Flermanian (1984).

\begin{tabular}{cc}
\hline Concordância & Classificação \\
\hline$<0,31$ & Nula \\
$0,31+0,51$ & Medíocre \\
$0,51+0,71$ & Moderada \\
$0,71+0,91$ & Boa \\
$0,91 \vdash 1,00$ & Excelente \\
\hline
\end{tabular}

Fonte: Autores (2021)

Figura 2. Concordância entre a idade real e a estimada de acordo com o dente e o sexo.

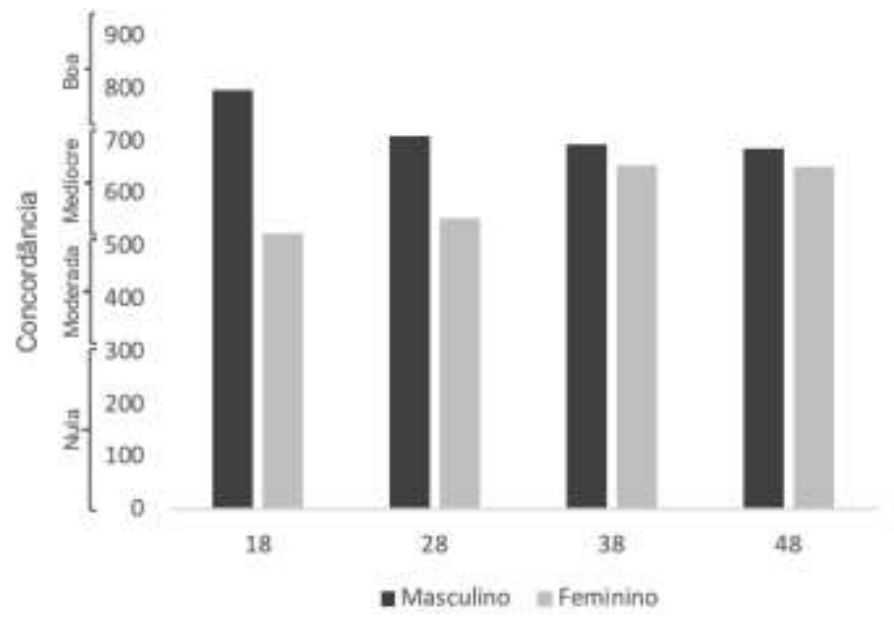

Fonte: Autores (2021)

Os gráficos de Bland e Altman, observados nas Figuras 3 e 4, são os que melhor representam os dados obtidos nos testes de Coeficiente de Correlação Intraclasse, onde quanto mais os pontos de dispersão estão próximos da linha média, mais eles concordam entre si. 
Research, Society and Development, v. 10, n. 7, e19410716524, 2021

(CC BY 4.0) | ISSN 2525-3409 | DOI: http://dx.doi.org/10.33448/rsd-v10i7.16524

Figura 3. Gráficos de Bland e Altman para o para o sexo masculino. A- dente 18; B- dente 28; C- dente 38 e D- dente 48.

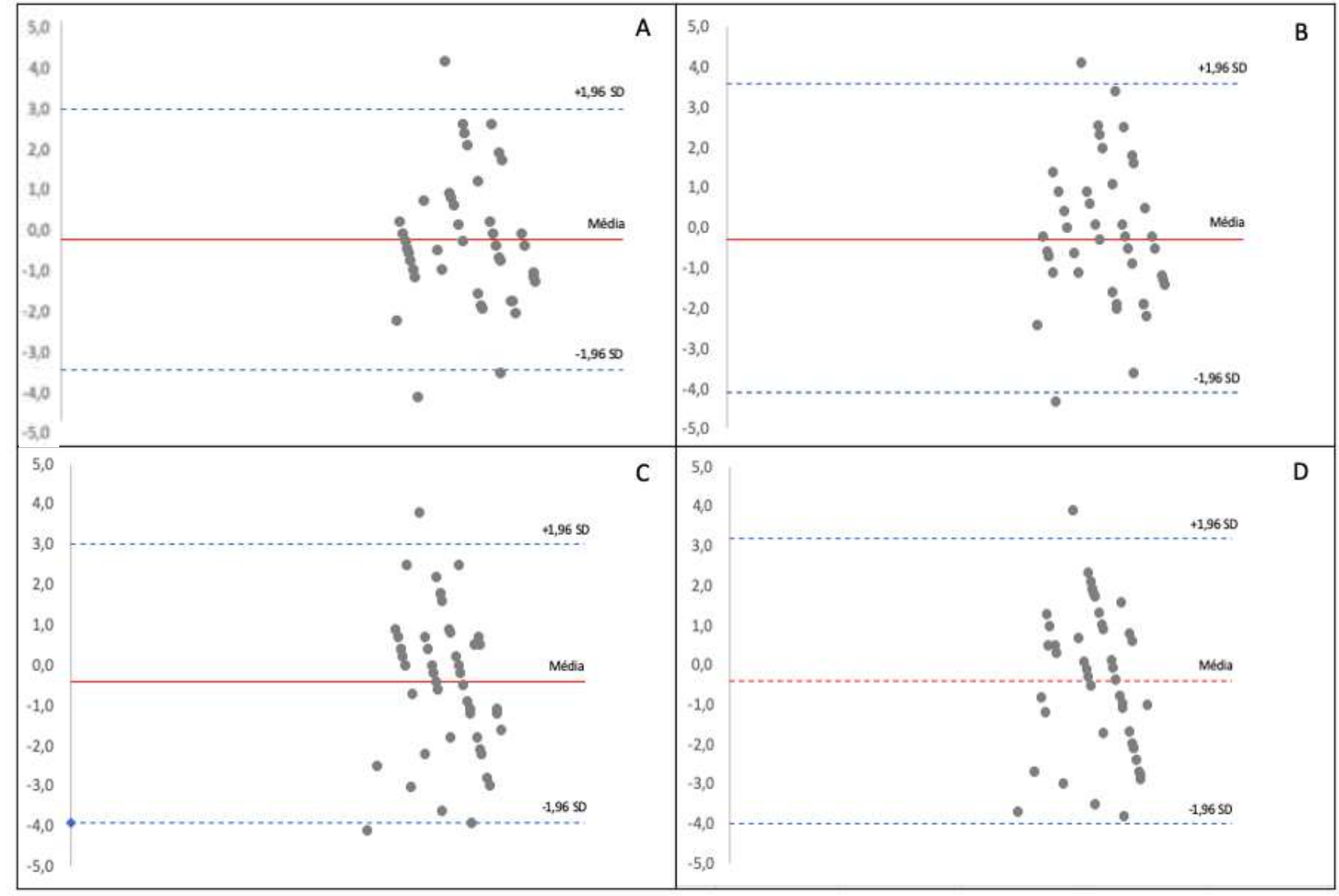

Fonte: Autores (2021). 
Figura 4. Gráficos de Bland e Altman para o para o sexo feminino. A- dente 18; B- dente 28; C- dente 38 e D- dente 48.

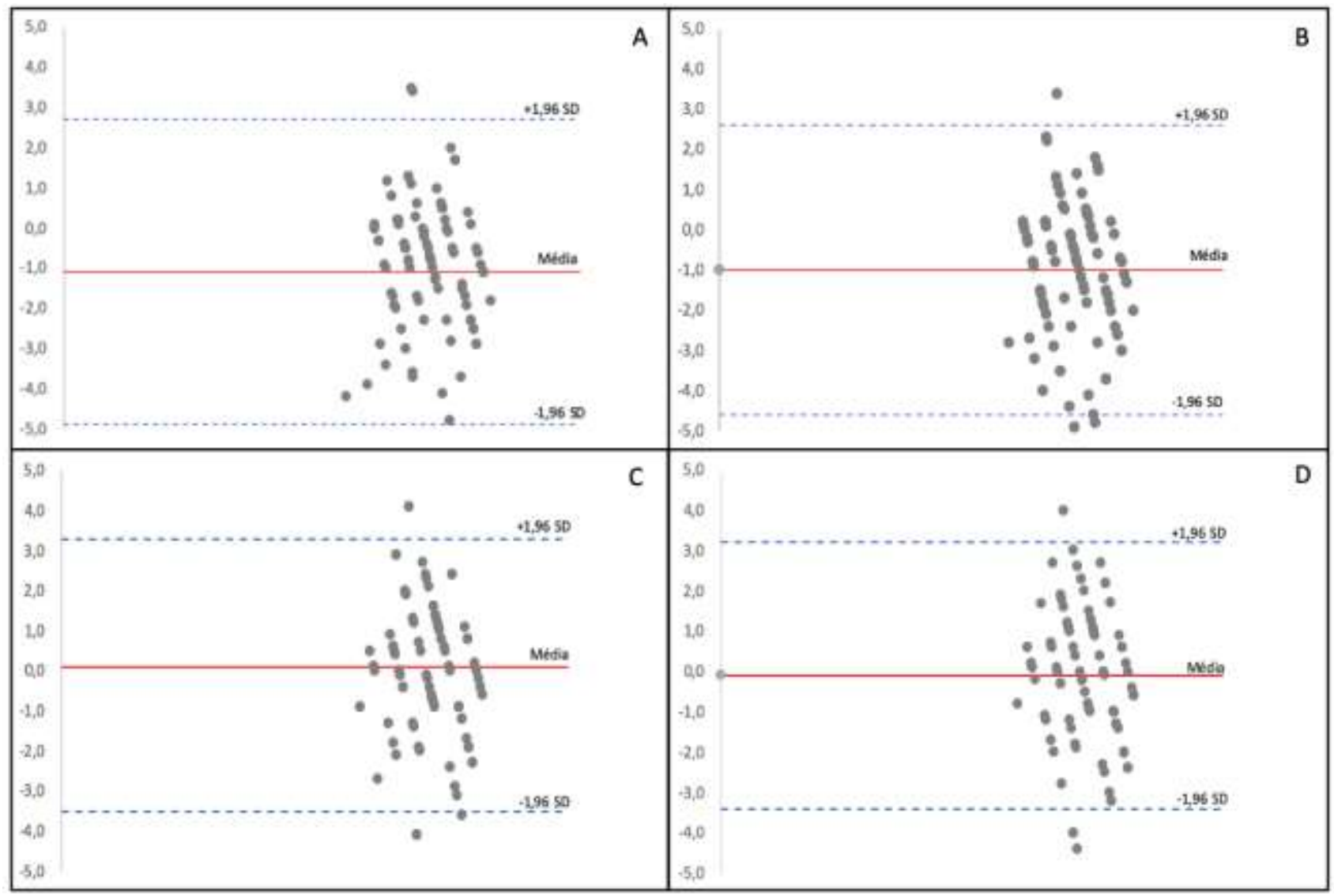

Fonte: Autores (2021).

\section{Discussão}

O processo de estimativa de idade através da arcada dentária possui muitas vantagens no meio forense, sendo de fácil aplicabilidade e com baixo custo na Odontologia. Dentre os métodos mais estudados na atualidade estão os propostos por Demirjian et al. em 1973 e o de Willems et al de 2001. O primeiro foi desenvolvido numa população franco-canadense a fim de determinar a maturidade dentária dos sete dentes permanentes da mandíbula, excluindo os terceiros molares. Alguns anos depois, o trabalho de Willems et al. (2001) revisou a técnica proposta por Demirjian et al. (1973) em uma população belga.

Neste estudo, há uma maior concordância entre a idade real e estimada no sexo masculino em detrimento do sexo feminino. Carvalho et al. (1990) avaliaram o desenvolvimento dos dentes permanentes de crianças brasileiras de Araçatuba SP utilizando o método proposto por Demirjian et al. (1973) também por radiografias panorâmicas, onde seus resultados mostram que o sexo feminino tem uma certa precocidade quanto à cronologia de mineralização dentária, o que pode interferir na estimativa de idade.

Nesta amostra, apesar da maioria dos elementos estudados serem considerados moderados de acordo com a classificação de Fermanian (1984), o elemento com melhor resultado foi o dente 18 no sexo masculino, classificado com boa concordância. Já no sexo feminino as melhores concordâncias foram entre os dentes 38 e 48, com resultados semelhantes. Considerando os terceiros molares superiores e inferiores como realizado neste estudo, Carneiro et al. (2010) desenvolveram uma tabela de cronologia de mineralização dos terceiros molares no Estado de Alagoas. Seus resultados mostraram uma 
correlação favorável entre a idade real e estimada da população amostral e asseguraram necessidade da criação de mais tabelas baseadas nas populações de cada região.

O presente estudo determinou a faixa etária entre 15 e 22 anos, com 33\% da amostra sendo do sexo masculino e $67 \%$ do sexo feminino. Sabe-se que o método de estimativa de idade torna-se mais confiável quando aplicado em indivíduos mais jovens devido aos estágios de mineralização distintos em que os terceiros molares se encontram (Gonçalves e Antunes, 1999). O estudo de Moreno et al. em 2014 buscou avaliar a aplicabilidade do método de Nicodemo, Moraes e Médici Filho na população da Paraíba - Brasil, utilizando uma amostra de indivíduos entre 10 e 25 anos, sendo o percentual de acertos da estimativa de idade maior entre aqueles que tinham entre 10 e 15 anos.

Diversos métodos de estimativa de idade foram desenvolvidos utilizando as radiografias. Neste estudo, as radiografias panorâmicas foram um recurso acessível, com boa visualização dos terceiros molares e sem grandes dificuldades na avaliação. Da mesma forma, Gruber e Kameyama (2001), em seu artigo de revisão, afirmam as vantagens das radiografias, por ser uma técnica não destrutiva e, além disso, os dentes humanos são estruturas que sofrem menos interferências de fatores ambientais e sistêmicos.

Em seu relato de caso, Rhonan et al. (2008) afirmam que além das características próprias de cada indivíduo, diversos fatores podem interferir no resultado da perícia, tais como fatores ambientais, sexuais, nutricionais, de hereditariedade e entre outros. Neste sentido, devido ao fato do Brasil ser um país de grande extensão territorial e de alta miscigenação, seria inviável aplicar uma única tabela de cronologia da mineralização baseada em indivíduos estrangeiros em uma população brasileira, esperando resultados acordantes, justificando a importância deste estudo com uma população nordestina.

Os primeiros estudos realizados com indivíduos brasileiros, foram realizados na região sudeste do país, como o de Nicodemo (1967), onde foi realizado um estudo da cronologia de mineralização dos terceiros molares baseado no exame radiográfico em indivíduos da região do Vale do Paraíba, no Estado de São Paulo, Brasil. A amostra foi determinada de acordo com fatores importantes como grupo étnico, clima, fatores socioeconômicos e naturalidade dos indivíduos. Em 1974, Nicodemo, Moraes e Medici Filho, após a conclusão de que nenhuma pesquisa se aplicava às características da população brasileira, utilizaram o método radiográfico para estimar a idade de 215 indivíduos a fim de elaborar, com confiabilidade, uma tabela de cronologia dos dentes permanentes de padrão nacional. Corroborando ao nosso estudo, os autores também não encontraram diferenças significativas entre os sexos masculino e feminino.

Tendo em vista que, a determinação da mineralização dos terceiros molares no universo forense é de extrema importância e que o presente estudo foi realizado com uma amostra nordestina, são necessárias mais pesquisas locais que determinem padrões específicos para cada região. Apesar do método de Cornélio Neto (2000) ter sido considerado neste trabalho como moderado para todos os terceiros molares, ele pode ser usado como um teste complementar para estimar a idade real de um indivíduo daquela região, necessitando de outros testes que corroborem com sua estimativa.

\section{Conclusão}

Após os resultados obtidos, pôde-se concluir que apesar da concordância apenas do dente 18 do sexo masculino ser considerada boa, a fórmula proposta por Cornélio-Neto (2000) é aceita como moderada para estimar a idade dos indivíduos de ambos os sexos da cidade de João Pessoa (PB). Portanto, recomenda-se o desenvolvimento de novos estudos e modificações na fórmula, que considerem as características da população regional.

\section{Referências}

Cameriere, R., Ferrante, L., \& Cingolani, M. (2006). Age estimation in children by measurement of open apices in teeth. International journal of legal medicine, 120(1), 49-52. 
Carneiro, A. P. C., Guimarães, J. A. T. L., Ikeda, R. M., Costa, A. P., Santiago, S., \& Laureano Filho, J. R. (2010). Chronological table of third molar mineralization in a survey in the state of Alagoas, Brazil. Brazilian journal of oral sciences, 9(4), 488-492.

Carvalho, A. A., de Carvalho, A., \& dos Santos Pinto, M. C. (1990). Estudio radiográfico do desenvolvimento da dentição permanente de crianças brasileiras com idade cronológica variando entre 84 e 131 meses. Revista de Odontologia da UNESP, 31-39.

Cornélio Neto, W. L. (2000). Estimativa da idade pela mineralização dentaria dos terceiros molares atraves de radiografias panoramicas.

Cornélio Neto, W. L., Conélio, G. C., \& Conceição, M. B. (2006). Estimativa da idade pela mineralização dentária dos $3^{\circ}$ molares através de radiografias panorâmicas. RGO-Rev Gaúcha Odontol, 54(3), 230-3.

Demirjian, A., Goldstein, H., \& Tanner, J. M. (1973). A new system of dental age assessment. Human biology, $211-227$.

Dhanjal, K. S., Bhardwaj, M. K., \& Liversidge, H. M. (2006). Reproducibility of radiographic stage assessment of third molars. Forensic science international, 159, S74-S77.

Fermanian, J. (1984). Mesure de l'accord entre deux juges: cas quantitatif. Rev Epidém et Santé Publ, 32, 408-413.

Gioster-Ramos, M. L., Silva, E. C. A., Nascimento, C. R., da Silva Fernandes, C. M., \& da Costa Serra, M. (2021). Técnicas de identificação humana em Odontologia Legal. Research, Society and Development, 10(3).

Gonçalves do Nascimento, L., Ribeiro Tinoco, R. L., Lacerda Protasio, A. P., Arrais Ribeiro, I. L., Marques Santiago, B., \& Cameriere, R. (2020). Age estimation in north east Brazilians by measurement of open apices. Journal of Forensic Odonto-Stomatology, 38(2).

Gruber, J., \& Kameyama, M. M. (2001). O papel da Radiologia em Odontologia Legal. Pesquisa Odontológica Brasileira, 15(3), 263-268.

Lee, S. H., Lee, J. Y., Park, H. K., \& Kim, Y. K. (2009). Development of third molars in Korean juveniles and adolescents. Forensic science international, 188(1-3), 107-111.

Moreno, M. B. P., Pontes, T. J. P., \& Rabello, P. M. (2014). Utilização da Tabela de cronologia de mineralização dental de Nicodemo, Moraes e Médici Filho na estimativa da idade de paraibanos. Saúde, Ética \& Justiça, 19(1), 35-44.

Nicodemo, R. A. (1967). Contribuição para o estudo da cronologia da mineralização dos terceiros molares, pelo método radiográfico, em leucodermas, brasileiros, residentes no Vale do Paraíba, Estado de São Paulo.

Nicodemo, R. A., Moraes, L. C., \& Médici Filho, E. (1974). Tabela cronológica da mineralização dos dentes permanentes entre brasileiros. Rev Fac Odontol São José dos Campos, 3(1), 55-6.

Nolla, C. M. (1960). The development of the human dentition. ASDC J Dent Child, 27(27), 254-266.

Oliveira, O. F. D., Fernandes, M. M., Daruge Júnior, E., Melani, R. F. H., \& Paranhos, L. R. (2010). Estimativa da idade por meio de radiografias panorâmicas. RGO. Revista Gaúcha de Odontologia (Online), 58(2), 203-206.

Orhan, K., Ozer, L., Orhan, A. I., Dogan, S., \& Paksoy, C. S. (2007). Radiographic evaluation of third molar development in relation to chronological age among Turkish children and youth. Forensic science international, 165(1), 46-51.

Silva, R. F., de Azevedo Marinho, D. E., de Lucena Botelho, T., Caria, P. H. F., Bérzin, F., \& Júnior, E. D. (2008). Estimativa da idade por meio de análise radiográfica dos dentes e da articulação do punho: relato de caso pericial. Arquivos em Odontologia, 44(2).

Santos Gonçalves, A. C., \& Antunes, J. L. F. (1998). Estimativa da idade em crianças baseada nos estágios de mineralização dos dentes permanentes, com finalidade odontolegal (Doctoral dissertation, Faculdade de Odontologia da Universidade de São Paulo na área de concentração em deodontologia e odontologia legal.)

Willems, G., Van Olmen, A., Spiessens, B., \& Carels, C. (2001). Dental age estimation in Belgian children: Demirjian's technique revisited. Journal of Forensic Science, 46(4), 893-895. 